\title{
Study of Carotid doppler in Patients with ischemic stroke.
}

\author{
Merina Gyawali ${ }^{1}$, Prakash Sharma ${ }^{2}$, Dipesh Karki ${ }^{3}$ \\ ${ }^{1}$ Assistant Professor, ${ }^{2}$ Associate Professor and Head, Department of Radio-diagnosis and Imaging, ${ }^{3}$ Assistant Professor, \\ Department of Medicine, Manipal College of Medical Sciences, Pokhara, Nepal.
}

Received: February 01, 2021

Accepted: February 20, 2021

Published: March 30, 2021

Cite this paper:

Gyawali M, Sharma P, Karki D. Study of Carotid doppler in Patients with ischemic stroke. Journal of Brain and Spine Foundation Nepal. 2021:2(1):24-30.

\section{Correspondence:}

Merina Gyawali

Assistant Professor

Department of Radio-diagnosis and Imaging

Manipal College of Medical Sciences, Pokhara, Nepal

Email: drmerinag@gmail.com

https://orcid.org/0000-0003-1025-1869.

\begin{abstract}
:
Introduction: Stroke is one of the major causes of increased morbidity and death. Large-vessel atherosclerosis of intracranial and extra cranial carotid vessels is an important cause of ischemic stroke. This research was undertaken to study the carotid Doppler findings in patients with acute ischemic stroke. Methods: A hospital-based prospective cross-sectional study was conducted from January 2020 till December 2020 in the department of Radio diagnosis and Imaging at Manipal Teaching Hospital, Pokhara, Nepal. Neuro-imaging and carotid Doppler findings in patients with ischemic stroke were studied. Data analysis was done using SPSS 20. Results: The mean age of subjects with ischemic stroke was $64 \pm 13.4$ years with the majority of cases in the age group of 51-70 years of age with male predominance ( $\mathrm{M}: \mathrm{F}=3: 2)$. Lacunar infarcts $(34.2 \%)$ were the most common findings followed by MCA infarct (30\%) and ACA infarct (10.8\%). Carotid plaques were seen in $43.3 \%$ patients. Bilateral ICA atheromatous plaque was seen in $48.1 \%$ of patients. Carotid bulb was the common site for plaque formation. Type III plaque was the commonest type. Significant ICA stenosis $>50 \%$ was observed in $24.2 \%$ patients. Age $>50$ years, male sex, smoking, hypertension, diabetes, and hyperlipidemia were important risk factors. Conclusion: Carotid artery Doppler demonstrated atherosclerotic plaques and significant stenosis in patients with ischemic stroke. Increasing age >50 years, male sex, smoking, hypertension, diabetes, and hyperlipidemia were associated with an increased rate of atherosclerosis, carotid stenosis and ischemic stroke.
\end{abstract}

Keywords: Atherosclerosis, Carotid artery, Doppler, Ischemia, Stenosis, Stroke

\section{Introduction:}

Stroke is a clinical syndrome of vascular origin, characterized by a sudden neurological deficit. ${ }^{1}$ It is associated with increased morbidity and death. Largevessel atherosclerosis of intracranial and extra cranial carotid vessels is an important cause of ischemic stroke. ${ }^{2}$

The risk factors in stroke are previous transient ischemic attack, history of diabetes, hypertension, dyslipidemia, presence of asymptomatic carotid plaques, and a high degree of carotid stenosis. ${ }^{3}$ The brain is supplied by four vessels: two internal carotid arteries and the two vertebral arteries. Obstructive disease, stenosis, ulcerative plaques, or anomalies anywhere in the cerebrovascular tree may produce a stroke or symptoms of cerebrovascular insufficiency. ${ }^{3,4}$

Doppler ultrasound is safe, non-invasive, cost-effective, and a very helpful modality in the examination of extra cranial carotid arteries. Carotid Doppler has largely replaced angiography for the detection of occlusive 
lesions in the vicinity of the carotid bifurcation and internal carotid artery. ${ }^{4,5}$ Carotid Doppler estimates the degree of stenosis, characterizes the plaque and identifies plaques with a high risk of embolization and intra-plaque hemorrhage. ${ }^{4}$ The accurate diagnosis of critical stenosis is important because these are the patients who carry an increased risk of cerebral infarction. ${ }^{4-6}$

The present research was undertaken to study the colour Doppler findings of extra cranial internal carotid arteries in patients presenting with ischemic stroke in a tertiary care center at Manipal Teaching Hospital in Pokhara, Gandaki Province, Nepal

\section{Methods:}

This observational, cross-sectional hospital-based prospective study was carried out in the Department of Radio diagnosis and Imaging at Manipal College of Medical Sciences and Teaching Hospital, Gandaki Province, Nepal from January 2020 till December 2020 for a duration of 12 months after obtaining ethical clearance from Institutional Research Committee (MEMG/IRC/335/GA) and informed consent from the patient or patient relatives.

The sample size was collected using the formula, $\mathrm{n}=4 \times \mathrm{p} \times \mathrm{q} / \mathrm{e} 2$

Where $\mathrm{p}=$ prevalence

$q=1-p$

$\mathrm{e}=$ margin of error $(0.05$ i.e. $5 \%)$

From a previous multicentre, large study comprising of 2096 patients, the prevalence of extra cranial Internal Carotid artery stenosis leading to ischemic stroke was $8.0 \%$. $^{7}$

So, sample size $\mathrm{n}=(4 \times 8 \times 92) / 25=117$
The calculated minimum sample size was 117. A total of 120 cases were taken over a period of 12 months which was the sample size adequate for the study.

All patients aged more than 18 years who presented with clinical features of stroke and showed evidence of no hemorrhage (i.e., ischemia) in CT / MRI were enrolled in the study. History, physical examination, and data considering demographic variables and clinical findings at presentation were collected. Risk factors such as hypertension, diabetes mellitus, dyslipidemia, smoking, including history of TIA or previous ischemic stroke were also documented.

All scans were performed by LOGIQ P3 by Wipro GE Ultrasound equipment using a high frequency $5-12 \mathrm{MHz}$ probe for evaluation of the extra cranial carotid arteries. The examination was performed after taking their informed consent. The study was carried out using realtime ultrasound equipment capable of B-mode imaging, pulsed-wave duplex scanning, and colour Doppler flow imaging and power Doppler imaging. The patient was placed in supine or semi-supine with head slightly hyper extended and rotated $45^{\circ}$ away from the side being examined. The scan was done in both transverse and longitudinal plane and common carotid artery, bulb, ECA, and ICA were examined bilaterally. On the carotid Doppler study, the studied parameters were Peak systolic velocity (PSV) of Internal carotid artery ICA, plaque characteristics, and grading of carotid artery stenosis (diameter stenosis). All the examinations were performed by the same operator with a Doppler angle of $<60^{\circ}$.

The patients underwent computed tomography (CT) scan and/or MRI of Brain prior to the color Doppler sonography of carotid arteries and various findings like the side of infarct (right/left), vascular territory (Middle cerebral artery/anterior cerebral artery/posterior cerebral 
artery) were noted. All consecutive patients with ischemic stroke were included in the study. Patients with haemorrhagic stroke, head injuries, other causes of cerebral edema, and space-occupying lesions in the brain, those with incomplete records or who fail to give informed consent were excluded from the study.

Data were collected covering all the relevant parameters for the study. All categorical data including findings on Carotid Doppler were expressed in percent and absolute number. All numerical continuous data were expressed in mean $\pm \mathrm{SD}$. Chi squared test was used to compare the significant difference of proportions for categorical data. All tests were analyzed with a $95 \%$ confidence interval and were considered statistically significant if $p$ value was $<0.05$. Statistical Packages for the Social Sciences (SPSS) 20 was used for data analysis.

\section{Results:}

A total of 201 patients presented with stroke and underwent CT or MRI in the department of Radio diagnosis and Imaging during the study period. One hundred and thirty-two patients with stroke were detected with non-haemorhage in CT/ MRI demonstrating that $65.7 \%$ of strokes were infarct or were of ischemic origin. Twelve patients were excluded from the study due to incomplete data and records. So, finally, 120 cases were taken up for the study. The study group comprised of $72(60 \%)$ males and 48 (40\%) females (M: $\mathrm{F}=3: 2$ ). The mean age of subjects was $64 \pm 13.4$ years with a range of $36-88$ years of age.

Patients were classified as per sex and age groups (Table 1). The majority $(\mathrm{n}=64 ; 53.3 \%)$ of cases were aged between 51-70 years of age. Nine (7.5\%) were young stroke patients, aged $<40$ years. There was no statistical significant difference in occurrence of stroke among two genders in different age groups $(\mathrm{p}=0.85 ; \mathrm{p}>0.05)$.
Table 1. Age groups/sex distribution $(\mathrm{n}=120)$

\begin{tabular}{lcccc}
\hline Sex & $\begin{array}{c}\leq 50 \\
\text { years }\end{array}$ & $\begin{array}{c}51-70 \\
\text { years }\end{array}$ & $\begin{array}{c}>71 \\
\text { years }\end{array}$ & Total \\
Male & 15 & 38 & 19 & 72 \\
Female & 8 & 26 & 14 & 48 \\
Total & 23 & 64 & 33 & 120
\end{tabular}

Conventional risk factors in the study participants were hypertension in $96(80 \%)$, diabetes in 75 (62.5\%), dyslipidemia in 62 (51.7\%), documented atrial fibrillation in 18 (15\%), and actively smoking in 104 $(86.6 \%)$. History of previous ischemic stroke was documented in $16(13.3 \%)$ patients.

Forty-six $(38.3 \%)$ patients presented with right sided hemiparesis, 41 (34.2\%) had left sided hemiparesis, 26 (21.7\%) had loss of consciousness, 21(17.5\%) had TIA and $12(10 \%)$ had history of ataxia and dizziness with no limb weakness. Motor aphasia was observed in 26 (21.7\%) patients while 21 (17.5\%) had facial deviation.

CT brain was done in all patients $(n=120 ; 100 \%)$. CT brain showed normal findings in 28 (23.3\%) cases. Additionally, MRI was done in 10 out of these 28 patients that demonstrated lacunars infarcts in 7 and posterior circulation infarcts in 3. Lacunar infarcts $(\mathrm{n}=41 ; 34.2 \%)$ were the most common findings in CT / MRI (Table 2).

Doppler ultrasonography demonstrated plaques in carotid vessels in 52 out of 120 (43.3\%) patients. Among the patients with atherosclerotic plaque, $69.1 \%$ were aged above 50 years, $85.4 \%$ had diabetes, $85.3 \%$ had hypertension and $79.1 \%$ had dyslipidemia. Around 75\% of them were active smokers. Strong association with stroke was observed with patients aged $>50$ years $(p=$ 0.05), diabetes $(\mathrm{p}<0.001)$, hypertension $(\mathrm{p}=0.01)$, 
dyslipidemia $(\mathrm{p}=0.001)$ and those with active smokers $(\mathrm{p}=0.03)$.

\begin{tabular}{lcc}
\hline Table 2: CT/ MRI brain findings $(\mathrm{n}=120)$ & \\
\hline Site/Type & Numbers & Percentage \\
Lacunar infarct & 41 & 34.2 \\
MCA infarct & 36 & 30 \\
ACA infarct & 13 & 10.8 \\
Posterior circulation & 12 & 10 \\
Normal findings & 18 & 15 \\
Total & 120 & 100 \\
\end{tabular}

Bilateral ICA atheromatous plaque was seen in 25 out of $52(48.1 \%)$ patients. Atheromatous plaques on the right ICA only was seen in $15(28.8 \%)$ patients and 12 (23.1\%) patients showed plaques only on the left ICA. So, a total of 77 ICA plaques were studied in 52 patients with carotid plaques.

Out of these 77 ICA plaques, 38 (49.3\%) were having plaques in the carotid bulb region, 25 (32.5\%) in the proximal part of the internal carotid artery, and 14 (18.2\%) patients in the distal part of the common carotid artery just proximal to the carotid bulb, demonstrating carotid bulb to be the common site for plaque formation. Majority of plaque were of low echogenicity 35 (45\%). The calcified plaque was present in 18 (23\%), moderately echogenic plaque in $5(7 \%)$ and hyper echogenic plaque was evident in 19 (25\%) subjects.

Sixty-eight $(56.7 \%)$ patients did not show plaque formation in extra cranial vessels but they had evidence of increased intima-media thickness. The mean intimamedia thickness was $0.92 \mathrm{~mm}$ among the stroke patients without plaque. It was $1.28 \mathrm{~mm}$ among the patients with carotid plaque.

Twenty-nine (24.2\%) patients with ischemic stroke had significant ICA stenoses $>50 \%$. Significant bilateral stenosis was not observed in any patients. Out of the 77 ICA plaques, 11 ICA showed $>230 \mathrm{~cm} / \mathrm{sec}$ peak systolic velocity at the site of stenosis caused by plaque formation accounting for more than $70 \%$ of stenosis, 18 had peak systolic velocity ranging from $125-230 \mathrm{~cm} / \mathrm{sec}$ accounting for more than $50 \%$ of stenosis and 48 had less than $125 \mathrm{~cm} / \mathrm{sec}$ showing less than $50 \%$ of stenosis (Table 3). Near-total occlusion was observed in 3 (2.5\%) cases.

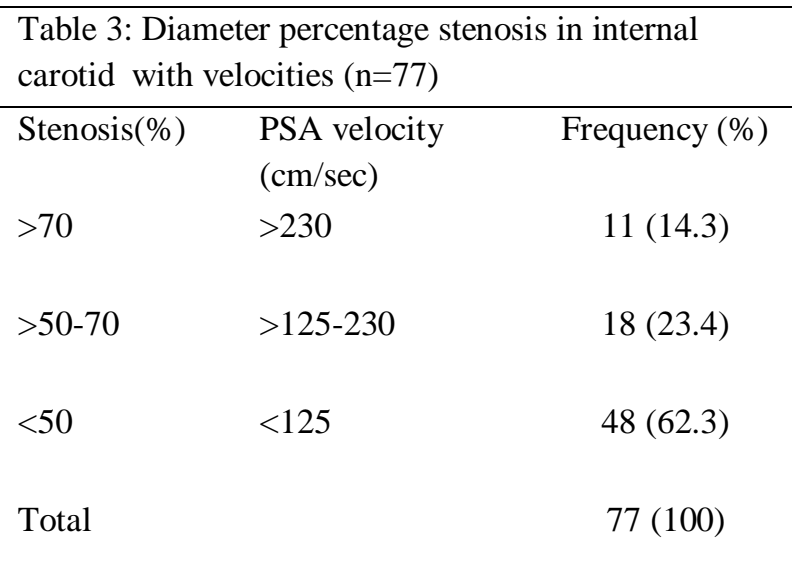

Most $(\mathrm{n}=37 ; 48 \%)$ of the atheromatous plaques were heterogeneously hyperechoic (type III). Uniformly hyperechoic (Type IV) comprised of 16 (20.8\%), type II (heterogeneously hypoechoic) accounted for 15 (19.5\%) and type I (uniformly hypoechoic) was least common $(n=9 ; 11.7 \%)$. In our study none of the patient showed plaque ulceration and hemorrhage.

\section{Discussion:}

The prevalence of ischemic stroke was $65.7 \%$ in the present study. It was almost similar $68 \%$ in the previous Nepalese study by Pathak et al. ${ }^{8}$ In our current study, the mean age of subjects with ischemic stroke was $64 \pm 13.4$ years with majority of cases in age group of 51-70 years 
of age with male predominance $(\mathrm{M}: \mathrm{F}=3: 2)$. The mean age of the patients was $61,60.5$, and 52 years with male dominance in an earlier study by Pathak et al. ${ }^{8}$, Syed et al. ${ }^{9}$, and Khan et al. ${ }^{10}$ In the studies by Fernandes et al. ${ }^{11}$ and Chamarthi et al. ${ }^{12}$, the highest incidence of stroke was found in the male population in the age group of 5170 years; findings consistent to our study. However, maximum number of patients belonged to even younger age group of 51-60 years according to Haq et al. $1^{3}$ About $7.5 \%$ were young stroke patients, aged $<40$ years in the current study. Even more, i.e., $12 \%$ patients were aged below 40 in the study by Bollipo et al. ${ }^{14}$

Conventional risk factors in the study participants were hypertension $(80 \%)$, diabetes $(62.5 \%)$, dyslipidemia $(51.7 \%)$ and actively smoking $(86.6 \%)$. These were the independent risk factors of ischemic stroke in previous published studies as well. ${ }^{8,11,15}$

The common clinical presentation of the study patients was right sided hemiparesis $(38.3 \%)$, left sided hemiparesis (34.2\%), motor aphasia (21.7\%), loss of consciousness $(21.7 \%)$, TIA (17.5\%) and facial deviation (17.5\%). Hemiparesis (predominantly right sided) followed by aphasia and loss of consciousness were similarly the commonest manifestation by Pathak et al. ${ }^{8}$, Fernandes et al. ${ }^{11}$, and Bollipo et al. ${ }^{14}$

No abnormal findings were seen in $15 \%$ in brain neuroimaging. Lacunar infarcts (34.2\%) were the most common findings followed by MCA infarct (30\%), ACA infarct $(10.8 \%)$ and posterior circulation infarct (10\%). Lacunar infarcts were similarly the most common CT findings according to Syed et al. ${ }^{9}$ and Fernandes et al. ${ }^{11}$

Doppler sonography diagnosed $43.3 \%$ patients to have plaques and stenosis within the carotid vessels. This was even higher; $70 \%, 78 \%$ and $82 \%$ according to Khan et al. ${ }^{10}$, Fernandes et al. ${ }^{11}$ and Bollipo et al. ${ }^{14}$
Among the patients with atherosclerotic plaque, $69.1 \%$ were aged above 50 years, $85.4 \%$ had diabetes, $85.3 \%$ had hypertension and 79.1\% had dyslipidaemia. Around $75 \%$ of them were active smokers in the current study. Increasing age, presence of hypertension, diabetes, dyslipidemia and smoking were significant independent factors for carotid atherosclerosis in the studies by Fernandes et al. ${ }^{11}$ Bollipo et al. ${ }^{14}$, Bharathi et al. ${ }^{15}$ and Yang et al. ${ }^{16}$

Bilateral ICA atheromatous plaques were seen in $48.1 \%$ patients in the current study. Bilateral plaques were observed in $31.7 \%, 35 \%, 46 \%$ and $59 \%$ according to Boliipo et al. ${ }^{14}$, Khan et al. ${ }^{10}$ Chamarthi et al. ${ }^{12}$ and Fernandes et al. ${ }^{11}$ respectively.

Out of the total 77 ICA plaques, $38(51.4 \%)$ were having plaques in carotid bulb region, $25(34.28 \%)$ in proximal part of internal carotid artery and 14 (14.28\%) patients in distal part of common carotid artery just proximal to carotid bulb in the current study. Carotid bulb was the common site for plaque formation in the current study as well as in the studies by Fernandes et al. ${ }^{11}$ and Chamarthi et al. ${ }^{12}$ In the current study, $56.7 \%$ patients did not show any plaque formation in extra cranial vessels, whereas it was $24 \%$ according to Chamarthi et al. ${ }^{12}$

Carotid intima-media thickness has been established as an independent predictor of coronary artery disease. ${ }^{17}$ Intima-media complex greater than $0.8 \mathrm{~mm}$ is considered as abnormal and represents the earliest change of atherosclerosis. An atheromatous carotid plaque is described as a focal increase in the IMT (more than 1.2 $\mathrm{mm}) .{ }^{17}$ Among the stroke patients without plaque, the mean intima media thickness was $0.92 \mathrm{~mm}$ in the current study whereas it was $1.07 \mathrm{~mm}$ according to Bollipo et al. ${ }^{14}$ The mean intima media thickness was $1.28 \mathrm{~mm}$ among the patients with carotid plaque in our study. It was even higher $(1.38 \mathrm{~mm})$ according to Bollipo et al. ${ }^{14}$ 
Significant ICA stenoses $>50 \%$ was observed in $24.2 \%$ patients with ischemic stroke in the current study. Similar (28\%) was the finding by Bharathi et al. ${ }^{15}$ It was however, lower (12\%) according to Chamarthi et al. ${ }^{12}$ ICA stenoses $>50 \%$ was observed higher (43\% and $45.1 \%$ ) in the studies by Khan et al. ${ }^{10}$ and Bollipo et al. ${ }^{14}$ respectively. Near total occlusion was seen in $2.5 \%$ of the patients while none of the patients had a complete occlusion in the current study. Similar were the findings by Bollipo et al. ${ }^{14}$ Significant bilateral stenosis was not observed in any patients in the current study, finding consistent with Khan et al. ${ }^{10}$, Fernandes et al. ${ }^{11}$ and Bharathi et al. ${ }^{15}$

Most commonly, 62.3\% ICA showed peak systolic velocity of less than $125 \mathrm{~cm} / \mathrm{sec}$ at the site of stenosis caused by plaque formation in the current study. It was $45 \%$ by Bollipo et al. ${ }^{14}$ In the current study, $14.3 \%$ ICA showed velocity of $>230 \mathrm{~cm} / \mathrm{sec}$ whereas, it was observed in $19 \%$ according to Bollipo et al. ${ }^{14}$

Most (48\%) of the atheromatous plaques were heterogeneously hyperechoic (type III) in the current study. Uniformly hyperechoic (Type IV) comprised of $20.8 \%$, type II (heterogeneously hypoechoic) accounted for $19.5 \%$ and type I (uniformly hypoechoic) was least common $(11.7 \%)$ in the present study. This was in consistency to the findings by Chamarthi et al. ${ }^{12}$ Type II (36\%) was the common morphological grading followed by type I (27\%) according to Bollipo et al. ${ }^{14}$ Whereas, majority of the plaques were Type I, uniformly hypoechoic (40\%), followed by Type IV calcified plaque (28\%) according to Garg et al. ${ }^{18}$

\section{Conclusions:}

Doppler sonography demonstrated that $43.3 \%$ patients with ischemic stroke had plaques involving carotid vessels. Significant ICA stenosis was observed in $24.2 \%$ patients. Increasing age $>50$ years, male sex, smoking, hypertension, diabetes and hyperlipidemia were associated with increased rate of atherosclerosis, carotid stenosis and ischemic stroke. Carotid Doppler is a very useful, non-invasive modality in detecting the site, morphology of atherosclerotic plaque, quantifying the degree of stenosis. Early detection of stenosis by carotid Doppler in high risk patients would be of great importance in managing and preventing cerebral ischemia or infarct.

\section{Reference:}

1. Donnan GA, Fisher M, Macleod M, Davis SM. Stroke. Lancet. 2008; 371(9624):1612-23. https://doi.org/10.1016/S0140-6736(08)60694-7.

2. Lovett JK, Coull AJ, Rothwell PM. Early risk of recurrence by subtype of ischemic stroke in populationbased studies. Neurology. 2004;62(4): 569-73. https://doi.org/10.1212/01.WNL.0000110311.09970.8 3.

3. Rothwell PM, Villagra R, Gibson R, Donders RC, Warlow CP. Evidence of a chronic systemic cause of instability of atherosclerotic plaques. Lancet. 2000; 355(9197):19-24. https://doi.org/10.1016/S01406736(99)04470-0.

4. Bluth EI. Evaluation and characterization of carotid plaque. Semin Ultrasound CT MR. 1997; 18(1):57-65. https://doi.org/10.1016/S0887-2171(97)90038-X.

5. Fontenelle LJ, Simper SC, Hanson TL. Carotid duplex scan versus angiography in evaluation of carotid artery disease. Am Surg. 1994; 60(110: 864-8. PMID: 7978683.

6. Bluth EI, Johnson SI, Troxclair L. The extra cranial cerebral vessels. In: Rummack CM, Levine D eds. Diagnostic Ultrasound. 5th ed. Philadelphia, USA: Elsevier; 2018. pp. 915-63.

7. Flaherty ML, Kissela B, Khoury JC, Alwell K, Moomaw CJ, Woo D, et al. Carotid artery stenosis as a cause of stroke. Neuroepidemiology. 2013; 40(1):3 641. https://doi.org/10.1159/000341410. 
8. Pathak V, Kanth R, Pant H. Stroke: a case series study in Nepal Medical College Teaching Hospital. Nepal Med Coll J. 2006; 8(3): 180-1. PMID: 17203825.

9. Syed NA, Khealani BA, Ali S, Hasan A, Akhtar N, Brohi $\mathrm{H}$, et al. Ischemic stroke subtypes in Pakistan: The Aga Khan University Stroke Data Bank. J Pak Med Assoc. 2003; 53(12): 584-8. Available from: https://jpma.org.pk/articledetails/1323?article_id=1323.

10. Khan SU, Lashari NA, Lakho NI, Faisal A, Hussain A. Cerebral ischaemia and stroke; Role of carotid Doppler. Professional Med J 2017; 24(12): 1823-7. https://doi.org/10.17957/TPMJ/17.4001.

11. Fernandes M, Keerthiraj B, Mahale AR, Kumar A, Dudekula A. Evaluation of carotid arteries in stroke patients using color doppler sonography: A prospective study conducted in a tertiary care hospital in South India. Int J Appl Basic Med Res. 2016; 6(1): 38-44. https://doi.org/10.17957/TPMJ/17.4001.

12. Chamarthi M, Lava Kumar B, Nirusha R, Pravallika I, Kejriwal GS. Color doppler evaluation of carotid arteries in stroke patients: a study conducted in a rural tertiary care medical college hospital in South India. IOSR Journal of Dental and Medical Sciences (IOSRJDMS). 2017; 16(1): 4-9. Available from: http://www.iosrjournals.org/iosr-jdms/papers/Vol16issue1/Version-11/B1601110409.pdf.

13. Haq S, Mathur M, Singh J, Kaur N, Sibia RS, Badhan R. Colour Doppler Evaluation of Extracranial Carotid Artery in Patients Presenting with Acute Ischemic Stroke and Correlation with Various Risk Factors. J Clin Diagn Res. 2017 Mar;11(3):TC01-TC05. https://doi.org/10.7860/JCDR/2017/25493.9541.

14. Bollipo JP, Rao PB. Color doppler assessment of extra cranial carotid arteries in carotid artery disease with correlation of risk factors in predicting cerebro vascular accident in patients with carotid atheromatous disease. Int J Adv Med. 2018; 5(6): 1402-6. https://doi.org/10.18203/2349-3933.ijam20184718.

15. Bharathi BM, Gullapalli R. A study on prevalence of carotid artery stenosis in acute ischaemic stroke patients in Amalapuram, Andhra Pradesh, India. J Res
Med Sci. 2019; 7(6): 2146-50. https://doi.org/10.18203/2320-6012.ijrms20192489.

16. Yang D, Iyer S, Gardener H, Della-Morte D, Crisby M, Dong $\mathrm{C}$, et al. Cigarette smoking and carotid plaque echodensity in the Northern Manhattan Study. Cerebrovasc Dis. 2015; 40(3-4):136-143. https://doi.org/10.1159/000434761.

17. Touboul PJ, Hennerici MG, Meairs S, Adams H, Amarenco P, Bornstein N, et al. Mannheim carotid intima-media thickness consensus (2004-2006). An update on behalf of the Advisory Board of the 3rd and 4th Watching the Risk Symposium, 13th and 15th European Stroke Conferences, Mannheim, Germany, 2004, and Brussels, Belgium, 2006. Cerebrovasc Dis. 2007; 23(1): 75-80. https://doi.org/10.1159/000097034.

18. Garg S, Kashikar SV, Phatak S. Colour doppler evaluation of extracranial carotid arteries: a clinical and radiological correlation. J Clin Diagn Res. 2016;10(1):TC06-TC10.

https://doi.org/10.7860/JCDR/2016/15426.7130. 